\title{
Prognostic Utility of Optical Coherence Tomography for Visual Outcome After Extended Endoscopic Endonasal Surgery for Adult Craniopharyngiomas
}

\section{Ning Qiao}

Beijing Tiantan Hospital, Capital Medical University

Chuzhong Li

Beijing Tiantan Hospital, Capital Medical University

Jing Xu

Beijing Tiantan Hospital, Capital Medical University

\section{Guofo Ma}

Beijing Tiantan Hospital, Capital Medical University

Jie Kang

Beijing Tiantan Hospital, Capital Medical University

\section{Lu Jin}

Beijing Tiantan Hospital, Capital Medical University

\section{Lei Cao}

Beijing Tiantan Hospital, Capital Medical University

Chunhui Liu

Beijing Tiantan Hospital, Capital Medical University

Yazhuo Zhang

Beijing Tiantan Hospital, Capital Medical University

Song-Bai Gui ( $\square$ guisongbai@yeah.net)

Beijing Tiantan Hospital, Capital Medical University https://orcid.org/0000-0002-3638-2018

\section{Research Article}

Keywords: Optical coherence tomography, Prognosis factors, Visual outcomes, Extended endoscopic endonasal surgery, Craniopharyngiomas

Posted Date: June 9th, 2021

DOl: https://doi.org/10.21203/rs.3.rs-574460/v1

License: (c) (1) This work is licensed under a Creative Commons Attribution 4.0 International License. Read Full License 
Version of Record: A version of this preprint was published at Frontiers in Oncology on January 6th, 2022. See the published version at https://doi.org/10.3389/fonc.2021.764582. 


\section{Abstract}

Optical coherence tomography (OCT) serving as a novel high resolution imaging technique can assess the retinal morphology. At present, a few studies are reported with limited evidence on the predictive value of OCT for visual outcome after optic nerve decompression. This study aims to utilize the largest series of adult craniopharyngiomas to evaluate the association between OCT parameters and visual outcome following the extended endoscopic endonasal surgery (EEES). From October 2018 to October 2020, one hundred and seventy eyes in 88 adult patients with craniopharyngiomas were retrospectively reviewed. Gross total resection was performed in $82(93.2 \%)$ patients. The median follow-up time was 10.9 months. Our study showed that increased temporal circumpapillary retinal nerve fiber layer (cpRNFL) thickness was associated with higher odds of visual acuity $(V A)$ improvement and maintenance ( $O R=1.070$; $P=0.035)$, and greater inferior $c p R N F L$ thickness was significantly associated with visual field (VF) improvement and maintenance $(\mathrm{OR}=1.034 ; \mathrm{P}=0.046)$. Tight adhesion was demonstrated as an independent adverse factor for either postoperative $\operatorname{VA}$ or $\mathrm{VF}(\mathrm{P}=0.048, \mathrm{P}=0.030$, respectively). Receiver operating characteristic (ROC) analysis further verified the robustness of the prediction model either in VA $(A U C=0.842 ; P<0.001)$ or $V F(A U C=0.849 ; P<0.001)$. Preoperative $O C T$ can effectively predict visual outcome after EEES for adult craniopharyngiomas. It can also serve as a reliable alternative to evaluate preoperative visual filed defects, especially for patients with lower compliance. Tight adhesion was confirmed as an independent risk factor for postoperative visual outcome. The OCT-based multivariable prediction models developed in present study may contribute to patient counseling on visual prognosis.

\section{Introduction}

Craniopharyngiomas are rare brain tumors originating from any point along the pituitary-hypothalamic axis, accounting for $1.2-4.6 \%$ of all intracranial tumors[25]. By reason of the close vicinity of optic chiasma, visual deterioration is known as a common complication following surgery for craniopharyngiomas $[6,34,29,4,20]$. Prognostic factors related to postoperative visual outcome, including age[2,14,8], symptoms duration[5], tumor size and volume[15], preoperative visual function[14], and optic atrophy[17], have been studied extensively, but results are not consistent.

Retrograde axonal degeneration caused by chronic optic nerves compression secondary to craniopharyngiomas often leads to thinner circumpapillary retinal nerve fiber layer (cpRNFL) and macular ganglion cell complex (mGCC), thus leading to irreversible visual dysfunction[28]. Hence, visual recovery largely relies on timely removal of optic nerves compression and the amount of viable axions[28,13]. Optical coherence tomography (OCT) can serve as a noninvasive in vivo method to quantitatively and objectively measure cpRNFL thickness and MGCC parameters $[33,28]$. The clinical efficiency of OCT as a predictor of visual recovery after surgery for pituitary adenomas, meningioma or pediatric craniopharyngiomas has already been verified[28,11,17,10,33,24,22,3]. Differing from pituitary tumors and meningioma, craniopharyngiomas often directly adhere to optic nerves, with a higher risk of postoperative visual deterioration. Compared with pediatric craniopharyngiomas, adult craniopharyngiomas more frequently cause visual impairment prior to surgery[25]. Therefore, 
investigating reliable predictive indicators of postoperative visual outcome may be beneficial for patients counseling on visual prognosis. However, there is limited evidence on the prognostic utility of OCT for visual outcome after surgery for adult craniopharyngiomas $[33,10]$.

This is first study to systematically evaluates the association between OCT parameters and visual outcome after the extended endoscopic endonasal surgery (EEES) for adult craniopharyngiomas.

\section{Materials And Methods}

\section{Patient Population}

From October 2018 to October 2020, a total of 118 adult patients underwent EEES for primary craniopharyngiomas at Beijing Tiantan Hospital of Capital Medical University. Inclusion criteria were as follows: (1) adult patients aged $\geq 18$ years, (2) newly confirmed diagnosis of craniopharyngioma, (3) computed tomography (CT), magnetic resonance imaging (MRI) and ophthalmologic tests before and after surgery. The exclusion criteria were: (1) past medical history of treatment including radiotherapy and surgery, (2) any ophthalmic condition other than compressive optic neuropathy caused by craniopharyngiomas, (3) any medical illness (including glaucoma, diabetes mellitus) known to affect optic apparatus, (4) ineligible OCT parameters, (5) unreliable visual field (VF) and best corrected visual acuity (BCVA) testing (fixation losses more than $20 \%$, false-negative error more than $20 \%$, and falsepositive error more than 20\%), (6) myopia greater than -6.00 diopters, (7) and papilledema on fundoscopy. Consequently, 88 (74.6\%) of 118 patients with primary craniopharyngiomas were retrospectively analyzed in this study. The flow chart for study inclusion and exclusion was described in Figure 1. All participants signed an informed consent form. The study was approved by the ethics committee of Beijing Tiantan Hospital of Capital Medical University.

\section{Radiological Evaluations}

The MRI examinations were performed preoperatively and at 3 and 9 months after surgery. Subsequent MRI scans were executed annually. Gross total removal (GTR) was defined as the resection without visual residual enhancing tumor according to postoperative MRI[20]. Tumor recurrence during follow-up was defined as the development of a pathological lesion on MRI that had not previously been observed or the regrowth of tumor residuals[20]. Tumor volume was calculated by using the following formula[21]: volume $=4 / 3 \pi(a / 2 \times b / 2 \times c / 2)$ (where $a, b$ and $c$ represent the diameters in the three dimensions).

\section{Visual Evaluation and Definition}

The ophthalmologic tests were performed preoperatively and at least 3 months after surgery. The BCVA was evaluated using logarithmic visual acuity chart, and then converted to the logarithm of the minimum angle of resolution (logMAR) for analysis. The VF examinations, including mean deviation (MD), pattern standard deviation (PSD) and visual field index (VFI), were performed using the Humphrey Field Analyzer (24-2 SITA-fast program, Carl Zeiss Meditec, Dublin, California, USA). OCT measurements, including 
cpRNFL thickness and mGCC parameters, were conducted using spectral-domain OCT (Optovue, Freemont CA, USA). OCT parameters were also analyzed based on decade of age: $£ 20$ years, 21-30 years, 31-40 years, 41-50 years, 51-60 years, and 61-70 years[16,12]. For the analysis, improvement or worsening in BCVA (normal ${ }^{3}$ 1.0) was defined as a change of greater than 0.1 in LogMAR visual acuity[19]. The VF improvement or worsening was defined as a change of $M D\left(\right.$ normal $^{3}-2$ decibels $(\mathrm{dB})$ ) greater than $-3 \mathrm{~dB}[26]$.

\section{Spectral-Domain Optical Coherence Tomography}

Subjects underwent SD-OCT scanning without pupillary dilation using Avanti RTVue-XR (Optovue, Fremont, California, USA) by experienced examiners on the same day as the ophthalmic evaluation. This equipment with an axial scan speed of $100 \mathrm{KHz}$ using an $840 \mathrm{~nm}$ wavelength laser has the resolution of $5.3 \mathrm{~mm}$ axially and $18 \mathrm{~mm}$ laterally. Three consecutive scans were performed on each eye. The scanning protocol for peripapillary RNFL thickness was acquired using the optic nerve head map, with a scanning range covering centered on the optic disc and covered a circle $3.45 \mathrm{~mm}$ in diameter. The GCC thickness was obtained using the GCC scanning protocol, which generates the data though the scans of a square grid $\left(7 \mathrm{~mm}^{\prime} 7 \mathrm{~mm}\right)$ on the central macula centered $1 \mathrm{~mm}$ temporal to the fovea and covered. Criteria for acceptable images included signal intensity level greater than 7 of 10 , signal strength index $\geq 40$. The normal RNFL and GCC thickness was defined as within the $95 \%$ percentile of age-, sex-, and race-matched normative values obtained from the manufacturer's database.

\section{Classification of Adhesion}

Compared to pituitary adenomas, craniopharyngiomas posed challenges mainly owing to their tendency to adhere to vital neurovascular structures, such as optic nerves and optic chiasma[30], with a higher risk of postoperative visual deterioration. Adhesion strength between optic apparatus and tumor was classified into two categories according to intraoperative findings by the surgeon: (1) no or loose adhesion if the tumor can be easily separated from the optic apparatus by gentle blunt dissection using dissectors; or (2) tight adhesion if the separation of the tumor required sharp dissection using scissors (Fig.2).

\section{Statistical Analysis}

We performed all statistical analyses with SPSS statistics software version 23 (IBM Corp). The data were presented as the mean \pm standard deviation (SD) or median (with interquartile range (IQR)) for normally distributed and nonnormally distributed samples, respectively. Differences between the pre- and postoperative visual outcomes were assessed by using the Wilcoxon signed rank test. Spearman's rank correlation coefficients were used to evaluate the relationship between OCT and VF parameters. The prognostic factors for visual outcome were analyzed by binary logistic regression. Receiver operating characteristic (ROC) curves were used to determine the performance of the prediction model. Area under the curve (AUC) with 95\% confidence interval $(\mathrm{Cl})$ and the associated P-value were calculated. $\mathrm{P}<0.05$ was considered statistically significant. 


\section{Results}

\section{Patient Characteristics}

The present cohort included 37 (42.0\%) male patients, and the mean age was 44.0 years old (range, 19-68 years). The most common preoperative symptom was visual impairment (78 patients; $88.6 \%$ ) and the mean duration of such symptom was 6.2 months (range, 1-24 months). The median tumor volume was $6.5 \mathrm{~cm}^{3}\left(\mathrm{IQR}, 3.4-14.0 \mathrm{~cm}^{3}\right)$. The clinicoradiological data of 88 patients was showed in Table 1.

\section{Preoperative Visual Function}

One hundred and twenty-three eyes (72.4\%) had VA impairment preoperatively. The median BCVA was 0.2 logMAR (IQR,0 to 0.5). VF defects occurred in one hundred and forty-nine eyes (87.6\%). MD, PSD, VFI on VF testing was -9.3 (IQR, -14.8 to -4.9), 7.7 (IQR, 3.5 -11.4), 77.5 (IQR, 56.5-90), respectively. The mean global RNFL thickness was $97.05 \pm 13.17 \mathrm{~mm}$. It was $121.72 \pm 19.36 \mathrm{~mm}$ in the inferior quadrant, 124.66 $\pm 20.24 \mathrm{~mm}$ in the superior quadrant, $70.53 \pm 13.96 \mathrm{~mm}$ in the nasal quadrant, and $70.28 \pm 12.42 \mathrm{~mm}$ in the temporal quadrant, respectively. Inner average, superior and inferior mGCC thickness was $91.68 \pm$ $9.15 \mathrm{~mm}, 91.00 \pm 9.34 \mathrm{~mm}$ and $92.36 \pm 9.49 \mathrm{~mm}$, respectively (Table 1). The associations between the mGCC parameters, cPRNFL thickness parameters and VF parameters in the 170 eyes were showed in Table 2. mGCC parameters significantly correlated with MD, PSD and VFI. All cpRNFL thickness parameters were significantly associated with MD except for the superior quadrant, PSD except for the nasal quadrant and VFI except for the inferior and nasal quadrant, respectively.

\section{Overall Surgical Results}

GTR was achieved in 82 (93.2\%) patients. Of the six cases with residual tumors, three were observed without further treatment, and three received gamma knife radiosurgeries postoperatively without causing new visual defects. Tight adhesion was observed in 31(35.2\%) patients. Adamantinomatous craniopharyngiomas were confirmed in $67(76.1 \%)$ patients. After a median follow-up duration of 10.9 months, recurrence occurred in $2(2.3 \%)$ patients. Of these patients, one did radiotherapy, and the other was observed without adjuvant therapy. There was no new visual impairment occurred in these two patients.

\section{Postoperative Visual Outcome}

The follow-up time was 10.9 (IQR, 7.2-16.2) months. Among 123 eyes with preoperative VA impairment, VA improved in $78.0 \%$ but worsened in $4.9 \%$ postoperatively. Five $(10.6 \%)$ of the 47 eyes with normal preoperative VA had postoperative VA deterioration. Of the 149 eyes with preoperative VF impairment, 83 (55.7\%) experienced improved or normalized VF, with no change in 58 (38.9\%), and 8 (5.4\%) experienced deterioration after surgery. Eighteen (85.7\%) of 21 eyes with normal preoperative VF showed no change and 2 (9.5\%) experienced worsening. The median BCVA after surgery was $0.1 \log M A R(I Q R, 0$ to 0.2$)$, which was significantly lower than the preoperative $0.2 \log M A R(I Q R, 0$ to 0.5$)(P<0.001)$. The MD (IQR) 
showed a significant improvement from -9.3 (IQR, -14.8 to -4.9) preoperatively to -5.3 (IQR, -9.9 to -2.5$)$ postoperatively $(\mathrm{P}<0.001)$.

\section{Prognostic Factors for Visual Prognosis}

Univariate logistic regression analysis for visual improvement and maintenance by OCT parameters were summarized in Table 3 , and increased temporal $(P=0.001)$ and inferior cpRNFL thickness $(P=0.004)$ proved to be independent prognostic factors. Clinicoradiological factors were also assessed, and the univariate analysis results revealed that tight adhesion and gender were associated significantly with postoperative visual outcome. In the multivariate analysis, increased temporal (OR, 1.070; 95\% confidence interval [CI], 1.005-1.140; $\mathrm{P}=0.035)$ and inferior cpRNFL thickness (OR,1.034; 95\% Cl,1.001-1.068; $\mathrm{P}=0.046$ ) proved to be independent favorable factors for VA (Fig. 3A and 4 ) and VF (Fig. 3B and 5 ) improvement and maintenance after surgery, respectively. Moreover, tight adhesion was confirmed as an independent risk factor for VA $(\mathrm{OR}, 0.188 ; 95 \% \mathrm{Cl}, 0.036-0.986 ; \mathrm{P}=0.048)$ or $\mathrm{VF}(\mathrm{OR}, 0.162 ; 95 \% \mathrm{Cl}, 0.032$ $0.836 ; \mathrm{P}=0.030$ ) after surgery for craniopharyngiomas. Multivariable prediction models developed for postoperative VA and VF recovery and maintenance, including age, gender, cpRNFL thickness and adhesion strength, showed AUC of $0.842(95 \% \mathrm{Cl}, 0.730-0.954 ; \mathrm{P}<0.001)$ and $0.849(95 \% \mathrm{Cl}, 0.741-0.958$; $P<0.001$ ), respectively (Fig. 6).

\section{Discussion}

Retrograde axonal degeneration resulting from chronic compression of optic chiasma can result in cpRNFL and mGCC thinning, consequently leading to irreversible visual impariment[28]. OCT allows quick, non-invasive, in vivo cross-sectional imaging of the retinal layers and it can serve as an important tool for objective quantification of $\mathrm{cpRNFL}$ and $\mathrm{mGCC}[10]$. There are growing evidences that preoperative OCT parameters are prognostic indicators of visual recovery after surgery for optic nerves decompression $[35,18,28,27,10]$. Danesh-Meyer et al[10]. first suggested RNFL thickness as a predictive factor following surgery for parachiasmal lesions. Park et al[28]. reported RNFL thickness was significantly associated with visual outcome following surgery for parachiasmal meningiomas. Mediero et al[24]. described that OCT may be a valid substitute when children were unable to perform perimetry and it can also predict visual outcome after surgery for pediatric craniopharyngiomas. Lee et al[22]. pointed out that the changes in retinal structure were closely related to peripapillary RNFL thinning and functional outcomes following surgery for pediatric craniopharyngiomas. Wang and his colleagues[33] demonstrated the predictive value of OCT parameters for visual outcome after pituitary tumor surgery. As far as we know, this is the first study to systematically analyze the prognostic utility of OCT parameters for visual outcome after extended endoscopic endonasal surgery for adult craniopharyngiomas.

In accordance with the results reported in previous studies[35,18,28,27,10], our research showed that preoperative OCT parameters can effectively predict postoperative visual outcome following optical nerves decompression. Notablely, increased inferior cpRNFL thickness was significantly associated with higher odds of VF recovery and maintenance $(P=0.046)$ in present study, which was consistent with the 
results reported in the earlier literature $[33,23]$. In addition, greater temporal cpRNFL thickness was confirmed as a significant favorable factor for VA recovery and maintenance $(P=0.035)$ in our study, which was similar to the findings by Kawaguchi and colleagues[19]. Furthermore, in our findings, the superonasal quadrant mGCL thicknesses $(P=0.091)$ and focal loss volume $(F L V)(P=0.087)$ showed statistical tendencies for visual recovery and maintenance. These tendencies were be in line with the findings declared in previous studies[35,27], although these tendencies were not significant. In our study, advancing age and gender failed to be predictors of visual outcome, in contrast to the results of previous studies $[2,17,9]$. However, in consideration of age-related changes and sexual difference in OCT parameters, age and gender still needed to be considered in multivariate analysis when using cpRNFL thickness to make clinical prediction models[12,16,1,32]. Moreover, the present study demonstrated that tight adhesion between craniopharyngioma and optic nevers was an independent risk factor for postoperative visual outcome, which was simlair to our previous results[31].Perhaps it was because tight adherence can predispose the optical nerves to mechanical and ischemic injury during the tumor resection. Overall, clinical prediction models established in the present study, incorporating age, gender, cPRNFL thickness and adhesion strength, suggested moderate discriminative abilities of VA $(A U C=0.842)$ and $\mathrm{VF}(\mathrm{AUC}=0.849)$ recovery and maintenance. It may be helpful to patient counseling on visual prognosis.

Operative trauma can be a confounder to postoperative visual outcome[28]. Compared with transcranial approaches[20], the extended endoscopic endonasal approach can provide a close-up view with better visualization of optic nerves and facilitate a lower visual deterioration after surgery[6,29,34], probably because there was less surgical trauma. Besides, this potential limitation was overcome by using the data of only one neurosurgeon (Songbai Gui). In addition, in our series, the mean follow-up time was 12.0 months (range, 3-28 months), which is longer than the period reported in Danesh-Meyer's series that the majority of visual recovery was inclined to happen within the first 6-10 weeks[11]. Considering the biological characteristic of craniopharyngiomas, the degree of the adhesion strength between optic nerves and tumor was evaluated according to intraoperative findings and included in multivariate analysis, which made it possible to control the possible confounding effect.

Our study demonstrated a statistically significant association between OCT parameters and MD/ PSD/ VFI, which is similar to the findings reported in Ohkubo's series[27] and Chung's series[7]. Therefore, OCT parameters can serve as an excellent alternative to assess preoperative visual field defects resulting from chronic chiasmal compression, particularly for patients with lower compliance.

\section{Limitation}

The single-centre setting and a retrospective study design have the potential to introduce selection bias, and our results required external validation in the future. In addition, detailed comparison between patients with normal and thin RNFL thickness about the extent of long-term visual recovery after surgery was limited by the present follow-up time, and serial ophthalmologic examinations should continue to be termly performed after surgery. 


\section{Conclusion}

Preoperative OCT proved to have an independent predictive value in visual outcome after extended endoscopic endonasal surgery for adult craniopharyngiomas. It can also serve as a reliable alternative to evaluate preoperative visual filed defects, especially for patient with lower compliance. Tight adhesion was also a strong predictor of postoperative visual outcome. The OCT-based multivariable prediction model developed in the current study may be beneficial to patient counseling on visual prognosis.

\section{Declarations}

Funding: The authors have no personal, financial, or institutional interest in any of the drugs, materials, or devices described in this article. This study is supported by the Beijing Municipal Science \& Technology Commission (Grant No. Z19110700660000).

Conflicts of interest/Competing interests: The authors declare that there is no conflict of interest.

Availability of data and material: Not applicable.

Code availability: Not applicable.

Ethics approval: The present study was approved by the Ethics Committee of Beijing Tiantan Hospital affiliated to Capital Medical University (Beijing, China).

Consent to participate: All patients were informed of the purpose of this study and signed a written consent form.

Consent for publication: The manuscript has not been published before and the manuscript is approved by all authors for publication.

Authors' contributions: All authors take responsibility for the integrity and the accuracy of this manuscript. Study concept and design: Qiao and Gui ; draft of the manuscript: Qiao, Li and Xu; acquisition of data: Qiao, Li, Ma, Xu, Kang and Jin ; statistical analysis: Qaio and Cao ; edit: Qiao; supervision: Liu and Zhang; revised: Qiao and Gui.

\section{Acknowledgments}

This study is supported by the Beijing Municipal Science \& Technology Commission (Grant No. Z19110700660000).

\section{References}

1. Adhi M, Aziz S, Muhammad K, Adhi MI (2012) Macular thickness by age and gender in healthy eyes using spectral domain optical coherence tomography. PloS one 7:e37638. 
doi:10.1371/journal.pone.0037638

2. Barzaghi LR, Medone M, Losa M, Bianchi S, Giovanelli M, Mortini P (2012) Prognostic factors of visual field improvement after trans-sphenoidal approach for pituitary macroadenomas: review of the literature and analysis by quantitative method. Neurosurgical review 35:369-378; discussion 378369. doi:10.1007/s10143-011-0365-y

3. Bialer OY, Goldenberg-Cohen N, Toledano H, Snir M, Michowiz S (2013) Retinal NFL thinning on OCT correlates with visual field loss in pediatric craniopharyngioma. Canadian journal of ophthalmology Journal canadien d'ophtalmologie 48:494-499. doi:10.1016/j.jcjo.2013.05.001

4. Campbell PG, McGettigan B, Luginbuhl A, Yadla S, Rosen M, Evans JJ (2010) Endocrinological and ophthalmological consequences of an initial endonasal endoscopic approach for resection of craniopharyngiomas. Neurosurg Focus 28:E8. doi:10.3171/2010.1.Focus09292

5. Carrim ZI, Reeks GA, Chohan AW, Dunn LT, Hadley DM (2007) Predicting impairment of central vision from dimensions of the optic chiasm in patients with pituitary adenoma. Acta Neurochir (Wien) 149:255-260; discussion 260. doi:10.1007/s00701-006-1108-7

6. Cavallo LM, Frank G, Cappabianca P, Solari D, Mazzatenta D, Villa A, Zoli M, D'Enza Al, Esposito F, Pasquini E (2014) The endoscopic endonasal approach for the management of craniopharyngiomas: a series of 103 patients. J Neurosurg 121:100-113. doi:10.3171/2014.3.Jns131521

7. Chung YS, Na M, Yoo J, Kim W, Jung IH, Moon JH, Lee J, Kim SH, Kim EH (2020) Optical Coherent Tomography Predicts Long-Term Visual Outcome of Pituitary Adenoma Surgery: New Perspectives From a 5-Year Follow-up Study. Neurosurgery 88:106-112. doi:10.1093/neuros/nyaa318

8. Cohen AR, Cooper PR, Kupersmith MJ, Flamm ES, Ransohoff J (1985) Visual recovery after transsphenoidal removal of pituitary adenomas. Neurosurgery 17:446-452. doi:10.1227/00006123198509000-00008

9. Danesh-Meyer HV, Carroll SC, Foroozan R, Savino PJ, Fan J, Jiang Y, Vander Hoorn S (2006) Relationship between retinal nerve fiber layer and visual field sensitivity as measured by optical coherence tomography in chiasmal compression. Investigative ophthalmology \& visual science 47:4827-4835. doi:10.1167/iovs.06-0327

10. Danesh-Meyer HV, Papchenko T, Savino PJ, Law A, Evans J, Gamble GD (2008) In vivo retinal nerve fiber layer thickness measured by optical coherence tomography predicts visual recovery after surgery for parachiasmal tumors. Investigative ophthalmology \& visual science 49:1879-1885. doi:10.1167/iovs.07-1127

11. Danesh-Meyer HV, Wong A, Papchenko T, Matheos K, Stylli S, Nichols A, Frampton C, Daniell M, Savino PJ, Kaye AH (2015) Optical coherence tomography predicts visual outcome for pituitary tumors. Journal of clinical neuroscience : official journal of the Neurosurgical Society of Australasia 22:1098-1104. doi:10.1016/j.jocn.2015.02.001

12. Duan XR, Liang YB, Friedman DS, Sun LP, Wong TY, Tao QS, Bao L, Wang NL, Wang JJ (2010) Normal macular thickness measurements using optical coherence tomography in healthy eyes of 
adult Chinese persons: the Handan Eye Study. Ophthalmology 117:1585-1594. doi:10.1016/j.ophtha.2009.12.036

13. Garcia T, Sanchez S, Litré CF, Radoi C, Delemer B, Rousseaux P, Ducasse A, Arndt C (2014) Prognostic value of retinal nerve fiber layer thickness for postoperative peripheral visual field recovery in optic chiasm compression. J Neurosurg 121:165-169. doi:10.3171/2014.2.Jns131767

14. Gnanalingham KK, Bhattacharjee S, Pennington R, Ng J, Mendoza N (2005) The time course of visual field recovery following transphenoidal surgery for pituitary adenomas: predictive factors for a good outcome. Journal of neurology, neurosurgery, and psychiatry 76:415-419. doi:10.1136/jnnp.2004.035576

15. Ho RW, Huang HM, Ho JT (2015) The influence of pituitary adenoma size on vision and visual outcomes after trans-sphenoidal adenectomy: a report of 78 cases. Journal of Korean Neurosurgical Society 57:23-31. doi:10.3340/jkns.2015.57.1.23

16. Huo YJ, Guo Y, Li L, Wang HZ, Wang YX, Thomas R, Wang NL (2018) Age-related changes in and determinants of macular ganglion cell-inner plexiform layer thickness in normal Chinese adults. Clinical \& experimental ophthalmology 46:400-406. doi:10.1111/ceo.13067

17. Jacob M, Raverot G, Jouanneau E, Borson-Chazot F, Perrin G, Rabilloud M, Tilikete C, Bernard M, Vighetto A (2009) Predicting visual outcome after treatment of pituitary adenomas with optical coherence tomography. Am J Ophthalmol 147:64-70.e62. doi:10.1016/j.ajo.2008.07.016

18. Jeon C, Park KA, Hong SD, Choi JW, Seol HJ, Nam DH, Lee JI, Shin HJ, Kong DS (2019) Clinical Efficacy of Optical Coherence Tomography to Predict the Visual Outcome After Endoscopic Endonasal Surgery for Suprasellar Tumors. World Neurosurg 132:e722-e731. doi:10.1016/j.wneu.2019.08.031

19. Kawaguchi T, Ogawa Y, Tominaga T (2019) Retinal Nerve Fiber Layer Thickness Measurement for Predicting Visual Outcome after Transsphenoidal Surgery: Optic Disc Atrophy Is Not the Deciding Indicator. World Neurosurg 127:e427-e435. doi:10.1016/j.wneu.2019.03.143

20. Kim YH, Kim CY, Kim JW, Kim YH, Han JH, Park CK, Paek SH, Oh CW, Kim DG, Jung HW (2012) Longitudinal analysis of visual outcomes after surgical treatment of adult craniopharyngiomas. Neurosurgery 71:715-721. doi:10.1227/NEU.0b013e318262146b

21. Lee DK, Sung MS, Park SW (2018) Factors Influencing Visual Field Recovery after Transsphenoidal Resection of a Pituitary Adenoma. Korean Journal of Ophthalmology 32. doi:10.3341/kjo.2017.0094

22. Lee GI, Park KA, Oh SY, Kong DS, Hong SD (2021) Inner and outer retinal layer thickness alterations in pediatric and juvenile craniopharyngioma. Sci Rep 11:2840. doi:10.1038/s41598-021-82107-5

23. Lee J, Kim SW, Kim DW, Shin JY, Choi M, Oh MC, Kim SM, Kim EH, Kim SH, Byeon SH (2016) Predictive model for recovery of visual field after surgery of pituitary adenoma. Journal of neurooncology 130:155-164. doi:10.1007/s11060-016-2227-5

24. Mediero S, Noval S, Bravo-Ljubetic L, Contreras I, Carceller F (2015) Visual Outcomes, Visual Fields, and Optical Coherence Tomography in Paediatric Craniopharyngioma. Neuroophthalmology 39:132139. doi:10.3109/01658107.2015.1039549 
25. Muller HL, Merchant TE, Warmuth-Metz M, Martinez-Barbera JP, Puget S (2019) Craniopharyngioma. Nat Rev Dis Primers 5:75. doi:10.1038/s41572-019-0125-9

26. Musch DC, Gillespie BW, Palmberg PF, Spaeth G, Niziol LM, Lichter PR (2014) Visual field improvement in the collaborative initial glaucoma treatment study. Am J Ophthalmol 158:96-104 e102. doi:10.1016/j.ajo.2014.04.003

27. Ohkubo S, Higashide T, Takeda H, Murotani E, Hayashi Y, Sugiyama K (2012) Relationship between macular ganglion cell complex parameters and visual field parameters after tumor resection in chiasmal compression. Japanese journal of ophthalmology 56:68-75. doi:10.1007/s10384-0110093-4

28. Park HH, Oh MC, Kim EH, Kim CY, Kim SH, Lee KS, Chang JH (2015) Use of optical coherence tomography to predict visual outcome in parachiasmal meningioma. J Neurosurg 123:1489-1499. doi:10.3171/2014.12.Jns141549

29. Park HR, Kshettry VR, Farrell CJ, Lee JM, Kim YH, Won TB, Han DH, Do H, Nyguist G, Rosen M, Kim DG, Evans JJ, Paek SH (2017) Clinical Outcome After Extended Endoscopic Endonasal Resection of Craniopharyngiomas: Two-Institution Experience. World Neurosurg 103:465-474. doi:10.1016/j.wneu.2017.04.047

30. Prieto R, Pascual JM, Rosdolsky M, Castro-Dufourny I, Carrasco R, Strauss S, Barrios L (2016) Craniopharyngioma adherence: a comprehensive topographical categorization and outcome-related risk stratification model based on the methodical examination of 500 tumors. Neurosurg Focus 41:E13. doi:10.3171/2016.9.Focus16304

31. Qiao N, Yang X, Li C, Ma G, Kang J, Liu C, Cao L, Zhang Y, Gui S (2021) The predictive value of intraoperative visual evoked potential for visual outcome after extended endoscopic endonasal surgery for adult craniopharyngioma. J Neurosurg:1-11. doi:10.3171/2020.10.Jns202779

32. Sabouri MR, Kazemnezhad E, Hafezi V (2016) Assessment of Macular Thickness in Healthy Eyes Using Cirrus HD-OCT: A Cross-Sectional Study. Medical hypothesis, discovery \& innovation ophthalmology journal 5:104-111

33. Wang MTM, King J, Symons RCA, Stylli SS, Meyer J, Daniell MD, Savino PJ, Kaye AH, Danesh-Meyer HV (2020) Prognostic Utility of Optical Coherence Tomography for Long-Term Visual Recovery Following Pituitary Tumor Surgery. Am J Ophthalmol 218:247-254. doi:10.1016/j.ajo.2020.06.004

34. Yamada S, Fukuhara N, Yamaguchi-Okada M, Nishioka H, Takeshita A, Takeuchi Y, Inoshita N, Ito J (2018) Therapeutic outcomes of transsphenoidal surgery in pediatric patients with craniopharyngiomas: a single-center study. Journal of neurosurgery Pediatrics 21:549-562. doi:10.3171/2017.10.Peds17254

35. Yoo YJ, Hwang JM, Yang HK, Joo JD, Kim YH, Kim CY (2020) Prognostic value of macular ganglion cell layer thickness for visual outcome in parasellar tumors. Journal of the neurological sciences 414:116823. doi:10.1016/j.jns.2020.116823

\section{Tables}


Table 1. Clinicoradiological Characteristics of the Enrolled 88 Patients 


\begin{tabular}{|c|c|}
\hline Parameters & Values, n (\%) \\
\hline Total number & 88 \\
\hline \multicolumn{2}{|l|}{ Sex } \\
\hline Male & $37(42)$ \\
\hline Female & $51(58)$ \\
\hline Age, y & $44.0 \pm 13.1$ \\
\hline \multicolumn{2}{|l|}{ Preoperative manifestations } \\
\hline Visual disturbance & $78(89)$ \\
\hline Menstrual disorder / impaired sexual function & $70(80)$ \\
\hline Headache & $58(66)$ \\
\hline Fatigue & $43(49)$ \\
\hline Polyuria/polydipsia & $33(38)$ \\
\hline \multicolumn{2}{|l|}{ Preoperative visual acuity } \\
\hline Normal & $23(26)$ \\
\hline Abnormal & $65(74)$ \\
\hline \multicolumn{2}{|l|}{ Preoperative visual field } \\
\hline No defect & $10(11)$ \\
\hline Defect & $78(89)$ \\
\hline \multicolumn{2}{|l|}{ Size of tumor } \\
\hline Volume (cm) & $6.5(\mathrm{IQR}, 3.4-14.0)$ \\
\hline \multicolumn{2}{|l|}{ Characteristics of tumor } \\
\hline Solid & $12(14)$ \\
\hline Cystic & $31(35)$ \\
\hline \multicolumn{2}{|l|}{ Solid and cystic } \\
\hline Cystic component $>50 \%$ & $29(33)$ \\
\hline Solid component $>50 \%$ & $16(18)$ \\
\hline With hydrocephalus & $33(37.5)$ \\
\hline With calcification & $49(55.7)$ \\
\hline Preoperative cpRNFL parameters (mm) & \\
\hline
\end{tabular}




\begin{tabular}{|l|l|}
\hline Average thickness & $97.05 \pm 13.17$ \\
\hline Superior quadrant & $124.66 \pm 20.24$ \\
\hline Inferior quadrant & $121.72 \pm 19.36$ \\
\hline Nasal quadrant & $70.53 \pm 13.96$ \\
\hline Temporal quadrant & $70.28 \pm 12.42$ \\
\hline Preoperative mGCC parameters $(\mathrm{mm})$ & \\
\hline Inner Average & $91.68 \pm 9.15$ \\
\hline Superior & $91.00 \pm 9.34$ \\
\hline Inferior & $92.36 \pm 9.49$ \\
\hline GCC FLV (\%) & $3.19 \pm 2.94$ \\
\hline GCC GLV $(\%)$ & $6.49 \pm 5.88$ \\
\hline
\end{tabular}

Values are presented as number (\%), mean \pm standard deviation, or median (with interquartile range $(\mathrm{IQR})$ ). cpRNFL=circumpapillary retinal nerve fiber layer; $\mathrm{mGCL}=$ macular ganglion cell layer; $\mathrm{GLV}=\mathrm{Global}$ loss volume; $\mathrm{FLV}=$ Focal loss volume

Table 2. Relationship between GCC parameters, RNFL thickness parameters and visual field parameters

\begin{tabular}{|c|c|c|c|c|c|c|}
\hline & MD & & PSD & & VFI & \\
\hline Variable & $r$ & $P$ value & $r$ & $P$ value & $r$ & $P$ value \\
\hline \multicolumn{7}{|l|}{ cpRNFL parameters } \\
\hline Average thickness & 0.226 & $0.003^{\star}$ & -0.342 & $<0.001^{\star}$ & 0.245 & $0.001^{*}$ \\
\hline Superior quadrant & 0.266 & $<0.001 *$ & -0.347 & $<0.001^{\star}$ & 0.288 & $<0.001^{\star}$ \\
\hline Inferior quadrant & 0.140 & 0.069 & -0.225 & $0.003^{*}$ & 0.124 & 0.106 \\
\hline Nasal quadrant & -0.039 & 0.611 & -0.086 & 0.266 & 0.003 & 0.971 \\
\hline Temporal quadrant & 0.327 & $<0.001^{*}$ & -0.356 & $<0.001^{*}$ & 0.325 & $<0.001^{\star}$ \\
\hline \multicolumn{7}{|l|}{ mGCC parameters } \\
\hline Inner Average & 0.264 & $0.001^{*}$ & -0.293 & $<0.001^{*}$ & 0.239 & $0.020 *$ \\
\hline Superior & 0.288 & $<0.001^{*}$ & -0.318 & $<0.001^{*}$ & 0.267 & $<0.001^{\star}$ \\
\hline Inferior & 0.235 & $0.002^{\star}$ & -0.259 & $<0.001^{\star}$ & 0.203 & $0.008^{*}$ \\
\hline Focal loss volume & -0.342 & $<0.001^{*}$ & 0.367 & $<0.001 *$ & -0.356 & $<0.001^{\star}$ \\
\hline Global loss volume & -0.332 & $<0.001^{*}$ & 0.334 & $<0.001^{*}$ & -0.317 & $<0.001^{*}$ \\
\hline
\end{tabular}


$\mathrm{cpRNFL=circumpapillary} \mathrm{retinal} \mathrm{nerve} \mathrm{fiber} \mathrm{layer;} \mathrm{mGCL}=$ macular ganglion cell layer; $\mathrm{MD}=$ mean deviation; $\mathrm{VFI}=$ visual field index; $\mathrm{PSD}=$ pattern standard deviation; The asterisk indicates statistical significance, $\mathrm{P}<0.05$

Table 3. Univariate logistic regression for visual improvement and maintenance by OCT parameters

\begin{tabular}{|lllll|}
\hline & \multicolumn{2}{l}{ VA improvement and maintenance } & \multicolumn{2}{l|}{ VF improvement and maintenance } \\
Variable & OR $(95 \% \mathrm{Cl})$ & P value & OR $(95 \% \mathrm{Cl})$ & P value \\
cpRNFL thickness $(\mathrm{mm})$ & & & & \\
\hline Average & $1.036(0.990-1.083)$ & 0.127 & $1.012(0.965-1.061)$ & 0.630 \\
\hline Superior & $1.012(0.982-1.043)$ & 0.440 & $0.990(0.960-1.021)$ & 0.509 \\
\hline Inferior & $1.019(0.989-1.051)$ & 0.216 & $1.049(1.016-1.083)$ & $0.004^{\star}$ \\
\hline Nasal & $1.020(0.976-1.066)$ & 0.375 & $1.018(0.972-1.066)$ & 0.444 \\
\hline Temporal & $1.104(1.041-1.172)$ & $0.001^{*}$ & $1.027(0.975-1.082)$ & 0.313 \\
\hline mGCC parameters (mm) & & & & \\
\hline Inner Average & $1.056(0.988-1.128)$ & 0.109 & $1.049(0.979-1.124)$ & 0.175 \\
\hline Superior & $1.056(0.991-1.126)$ & 0.091 & $1.041(0.974-1.112)$ & 0.235 \\
\hline Inferior & $1.046(0.983-1.113)$ & 0.152 & $1.049(0.983-1.119)$ & 0.146 \\
\hline GCC FLV (\%) & $0.860(0.723-1.022)$ & 0.087 & $0.958(0.781-1.175)$ & 0.681 \\
\hline GCC GLV (\%) & $0.933(0.855-1.019)$ & 0.122 & $0.950(0.865-1.044)$ & 0.290 \\
\hline
\end{tabular}

$\mathrm{OCT}=$ optical coherence tomography; $\mathrm{VA}=\mathrm{Visual}$ acuity; $\mathrm{VF}=\mathrm{Visual}$ field; $\mathrm{BCVA}=$ Best corrected visual acuity; $M D=$ mean deviation; $c p R N F L=$ circumpapillary retinal nerve fiber layer; $m G C L=$ macular ganglion cell layer; GLV=Global loss volume; FLV=Focal loss volume; The asterisk indicates statistical significance, $\mathrm{P}<0.05$

\section{Figures}




\section{Enrollment}

Excluded $(n=30)$

- Diabetes mellitus ( $n=7)$

- Patients with any incomplete or missing data $(n=21)$

- Patients with disorders of consciousness failed to complete preoperative ophthalmologic examination $(n=2)$

Eyes excluded with reasion: OCT parameters and unreliable VF results $(n=6)$

Eyes included in analyses

$(n=170)$

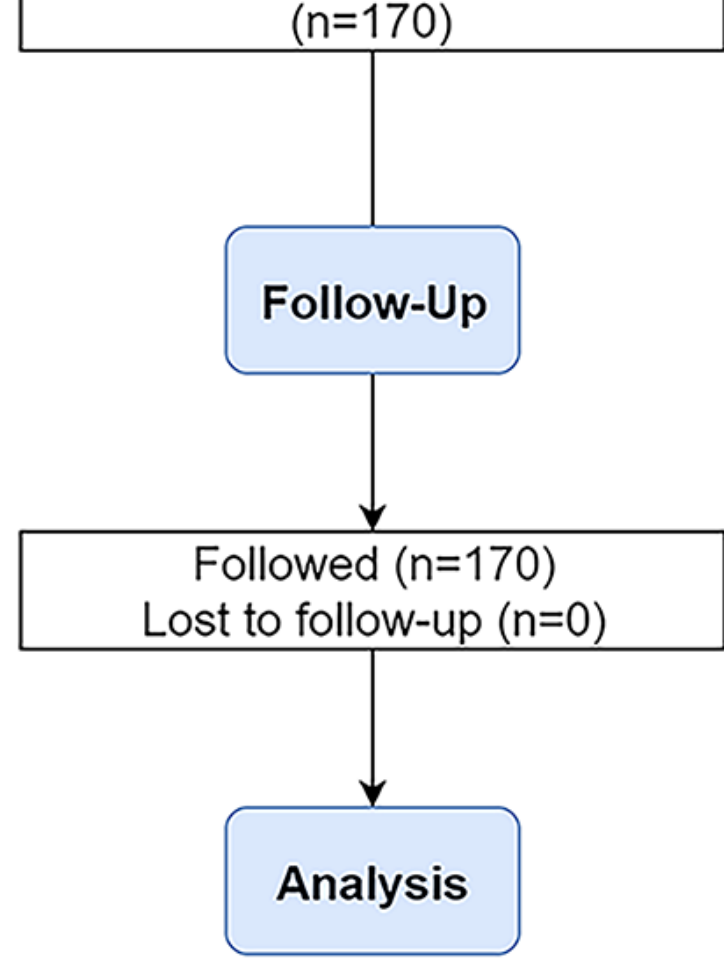

Figure 1

Flow chart demonstrating the inclusion/exclusion criteria used in the selection process. OCT=optical coherence tomography; $\mathrm{VF}=\mathrm{visual}$ field. 


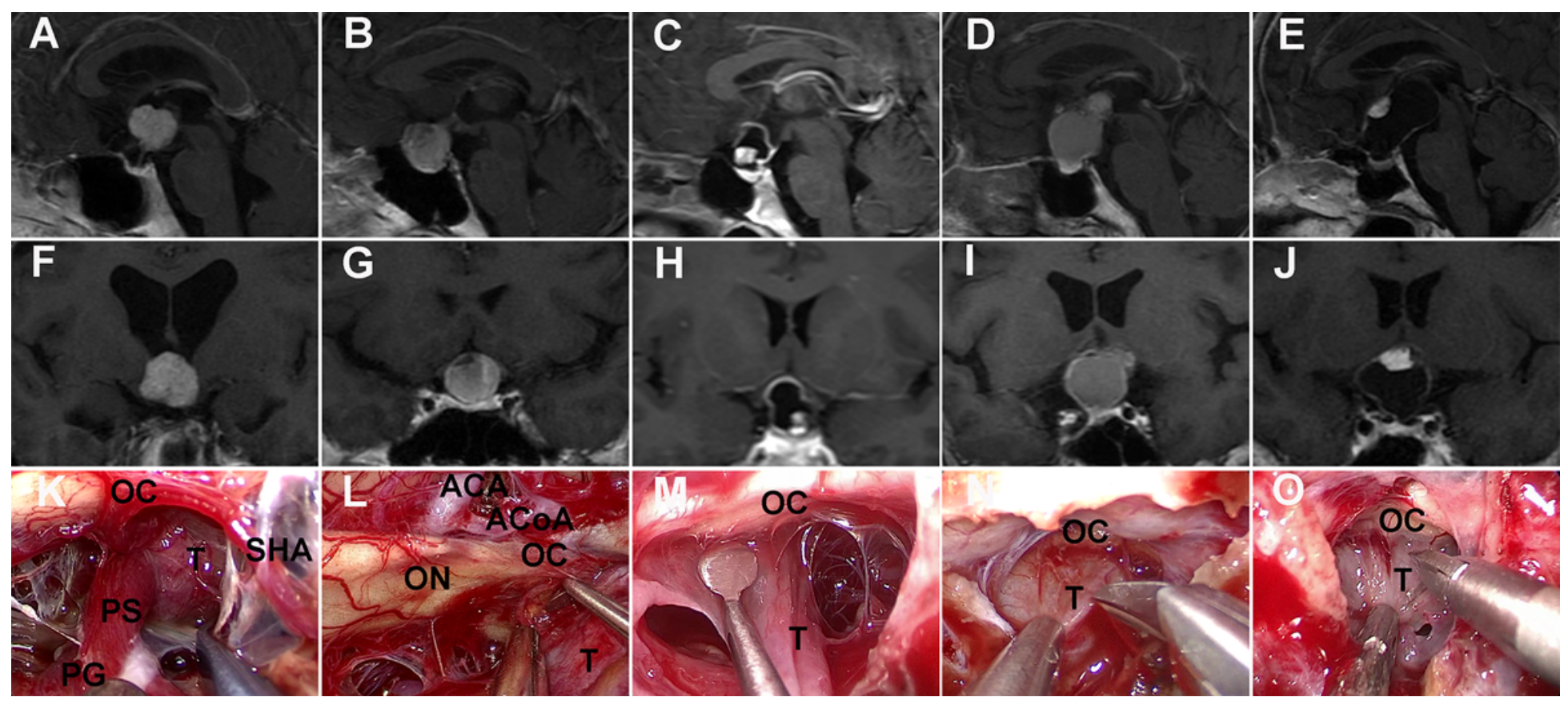

Figure 2

Adhesion strength between craniopharyngioma and optic nerves was intraoperatively evaluated. $\mathrm{K}$, Land M: No or loose adhesion. Contrast-enhanced T1-weighted MRI scans (A, F) showing an intrinsic third ventricular solid tumor compressing forward the optic chiasm. Intraoperative view $(K)$ revealing that the proximal part of the pituitary stalk extending from the gland could be identified as intact and at a normal size before tumor resection. Preoperative MRI scans (B, C, G, H) showing a sellar-suprasellar /suprasellar cystic-solid tumor stretching upward the optic chiasm. Surgical view $(L, M)$ showing the tumor can be easily separated from the optic nerves by dissector. $\mathrm{N}$ and $\mathrm{O}$ : Tight adhesion. Preoperative MRI scans (D, $\mathrm{E}, \mathrm{I}, \mathrm{J}$ ) showing sellar-suprasellar cystic-solid tumors displacing the optic chiasm. Intraoperative videocaptured photographs $(\mathrm{N}, \mathrm{O})$ showing tight adhesion between the tumor and the optic apparatus needing sharp dissection using scissors. $\mathrm{OC}=$ optic chiasma; $\mathrm{ON}=$ optic nerve; $\mathrm{T}=$ tumor; $\mathrm{PG}=$ pituitary gland; $\mathrm{PS}=$ pituitary stalk; $\mathrm{SHA}=$ superior hypophysial artery; $\mathrm{ACA}=$ anterior cerebral artery; $\mathrm{ACo} \mathrm{A}=$ anterior communicating artery. 
A

Increased temporal cpRNFL thickness

Preoperative BCVA

Tight adhesion

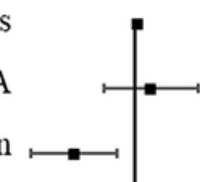

$1.104(1.041-1.172) \quad 0.001 *$

$1.070(1.005-1.140) \quad 0.035^{*}$

Tight adhesion

Duration of symptom, per 1 month increase

$1.820(0.328-10.103) \quad 0.493$

$0.108(0.023-0.518)$

$0.005 *$

$0.188(0.036-0.986) \quad 0.048 *$

Larger volume

$0.964(0.883-1.051) \quad 0.403$

Male

$0.975(0.944-1.006) \quad 0.109$

$0.295(0.075-1.153) \quad 0.079$

Age, per 10 years

Adamantinomatous craniopharyngiomas

$1.112(0.698-1.772) \quad 0.654$

$0.298(0.037-2.397) \quad 0.255$

$0.283(0.059-1.350) \quad 0.113$

Calcification

Cystic component $>50 \%$

Hydrocephalus

Gross total resection

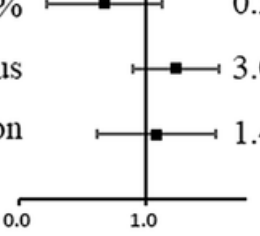

$0.224(0.028-1.802) \quad 0.160$

$3.032(0.634-14.493) \quad 0.165$

$1.490(0.173-12.830) \quad 0.717$

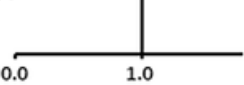

B

Increased inferior $\mathrm{cpRNFL}$ thickness

Preoperative $\mathrm{MD}$

Tight adhesion

Duration of symptom, per 1 month increase

Larger volume

Male

Age, per 10 years

Adamantinomatous craniopharyngiomas

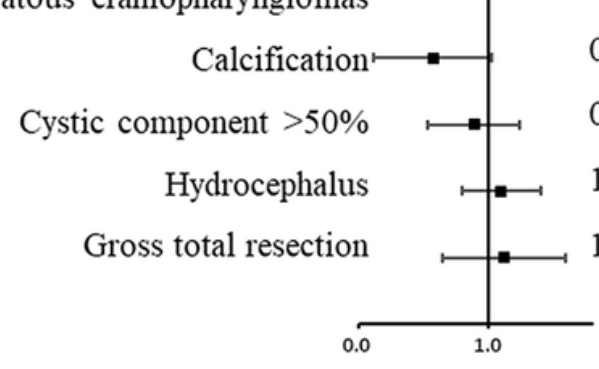

$1.049(1.016-1.083) \quad 0.004 *$

$0.939(0.841-1.048) \quad 0.260$

$0.162(0.032-0.836)$

$1.128(0.926-1.375) \quad 0.232$

$0.978(0.946-1.012) \quad 0.201$

$0.194(0.040-0.945) \quad 0.042 \% \longmapsto$

$1.282(0.779-2.111) \quad 0.328$

$0.776(0.158-3.807) \quad 0.754$

$0.139(0.017-1.125) \quad 0.064$

$0.583(0.119-2.849) \quad 0.505$

$1.515(0.378-6.079) \quad 0.558$

$1.667(0.192-14.494) 0.643$
$1.034(1.001-1.068) \quad 0.046 *$

$0.162(0.032-0.836) \quad 0.030 *$

$0.300(0.057-1.585) \quad 0.156$

$1.207(0.698-2.089) \quad 0.501$

\section{Figure 3}

Univariate and multivariate logistic regression analyses were used to evaluate the predictive factors for visual prognosis following surgery for craniopharyngiomas. The black squares indicate the OR values, error bars represent $95 \% \mathrm{Cls}$, and $* \mathrm{P}<0.05$. According to the analysis, increased temporal $(\mathrm{A})$ and inferior (B) CpRNFL were favorable factors for postoperative viusal acuity and viusal field, respectively. Tight adhesion was an adverse factor for viusal revovery. $c p R N F=$ circumpapillary retinal nerve fiber layer; $B C V A=$ best corrected visual acuity; $M D=$ mean deviation. 

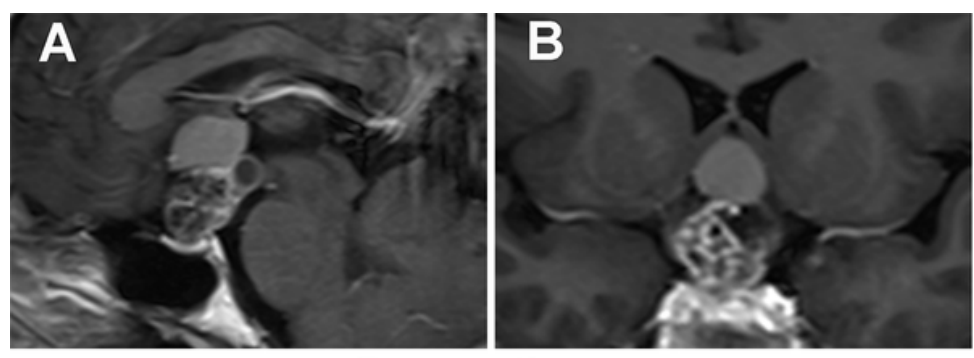

Preoperative

OS

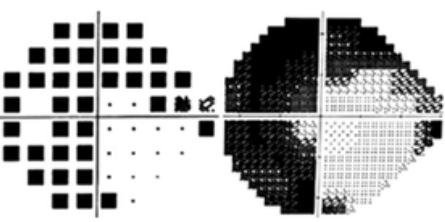

$B C V A=0.5 \quad M D=-17.18 d B$

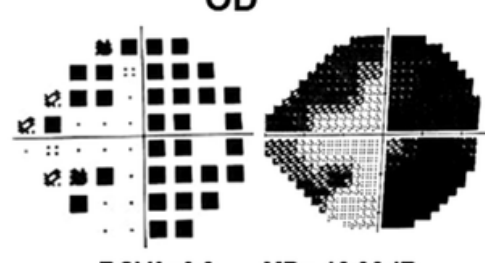

$B C V A=0.8 \quad M D=-18.98 \mathrm{~dB}$

RNFL Analysis
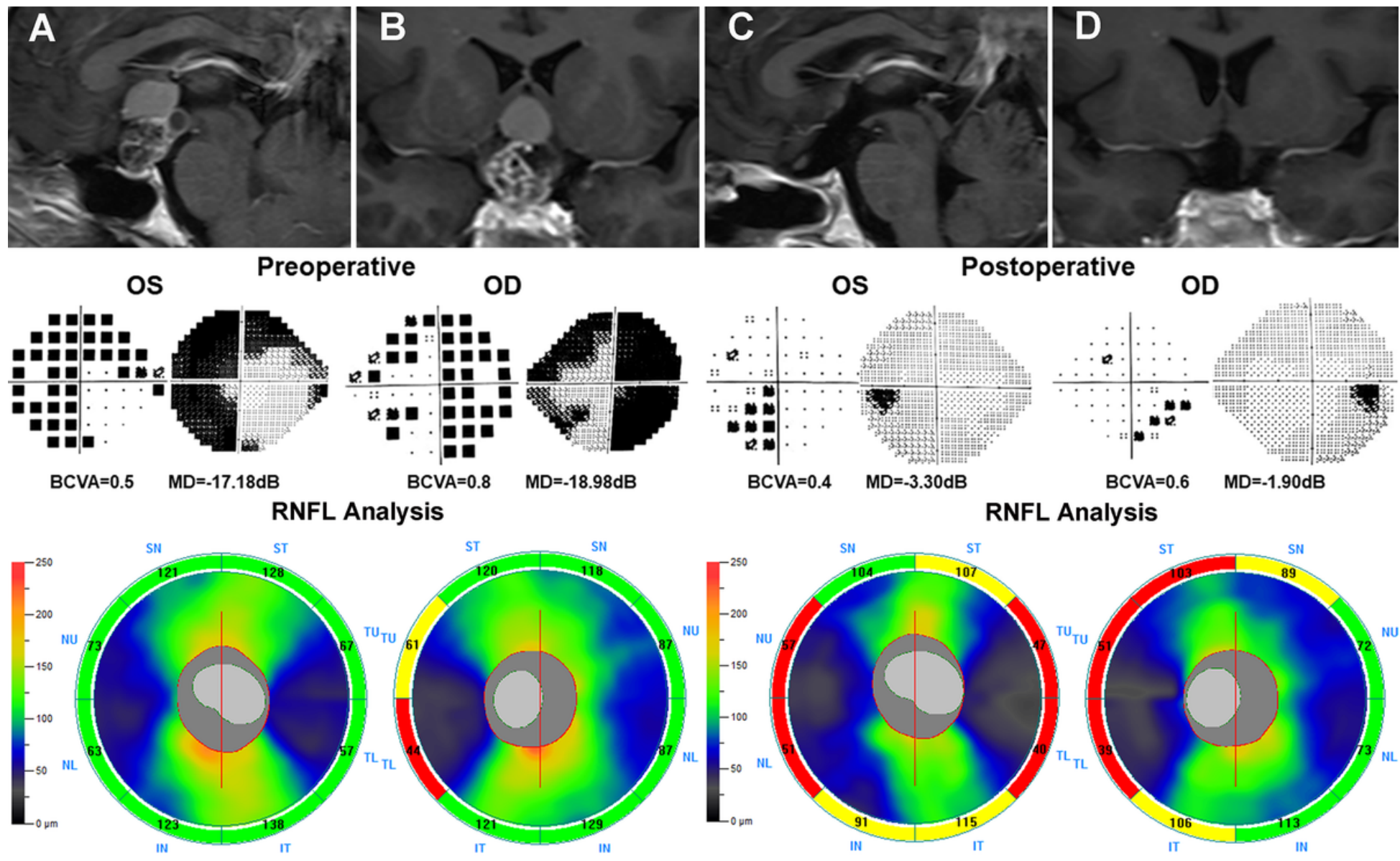

Postoperative

OS

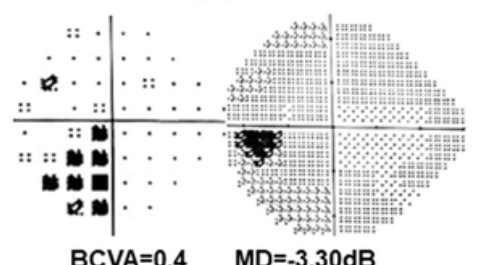

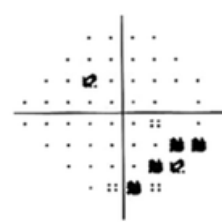

$B C V A=0.6 \quad M D=-1.90 \mathrm{~dB}$

\section{RNFL Analysis}
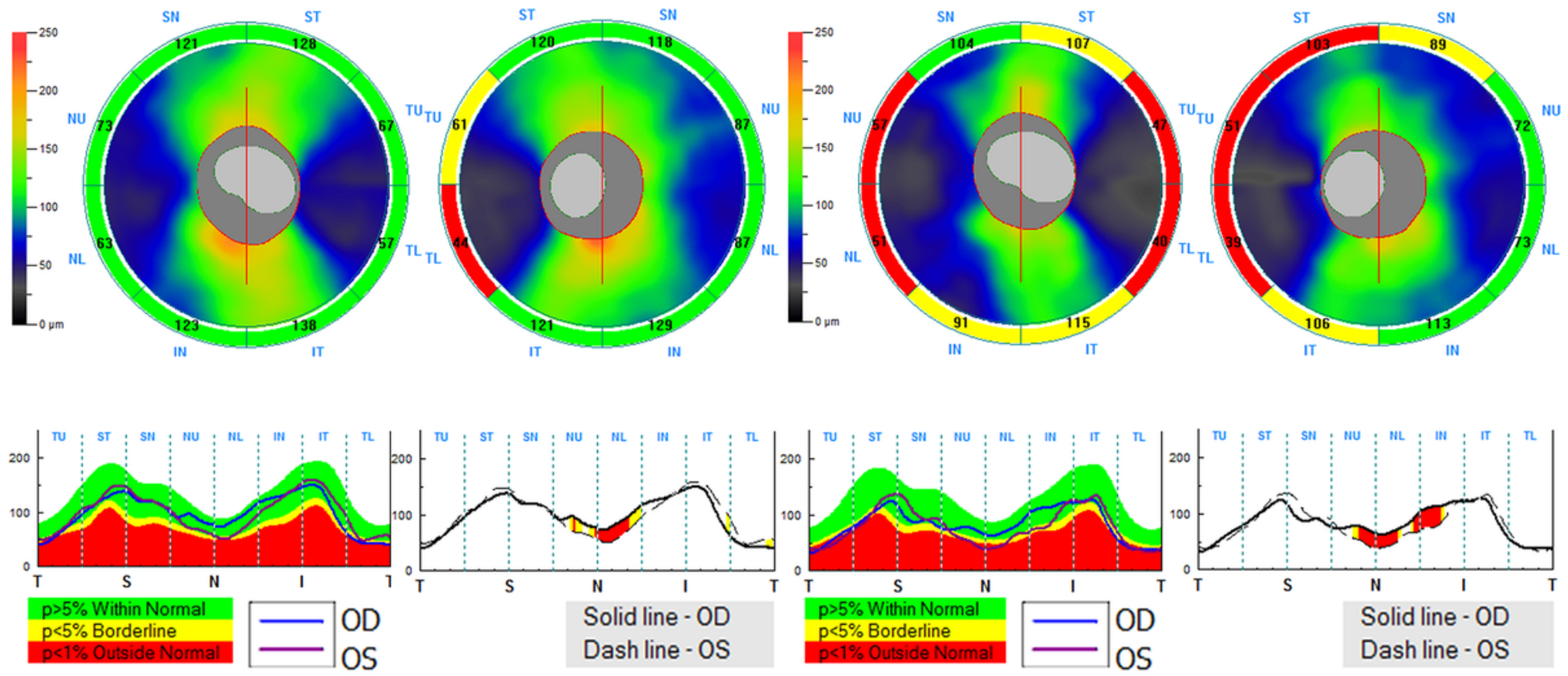

Figure 4

A 46-year-old female patient who underwent extended endoscopic endonasal surgery for

craniopharyngiomas was evaluated before and three months after surgery. Preoperative MRI scans (A, B) reveals a suprasellar cystic-solid tumor involving the third ventricle compressing the optic chiasma.

Preoperative visual field test showed mostly temporal visual field defects in both eyes. Preoperative OCT demonstrated decreased cpRNFL temporal thickness in right eye. Total removal of the tumor (C, D) led to the dramatic improvement of visual field and deterioration of visual acuity in both eyes confirmed by postoperative phthalmologic examination. $\mathrm{OCT}=0$ ptical coherence tomography; $\mathrm{cpRNF}=$ circumpapillary retinal nerve fiber layer; $B C V A=$ best corrected visual acuity; $M D=$ mean deviation. 

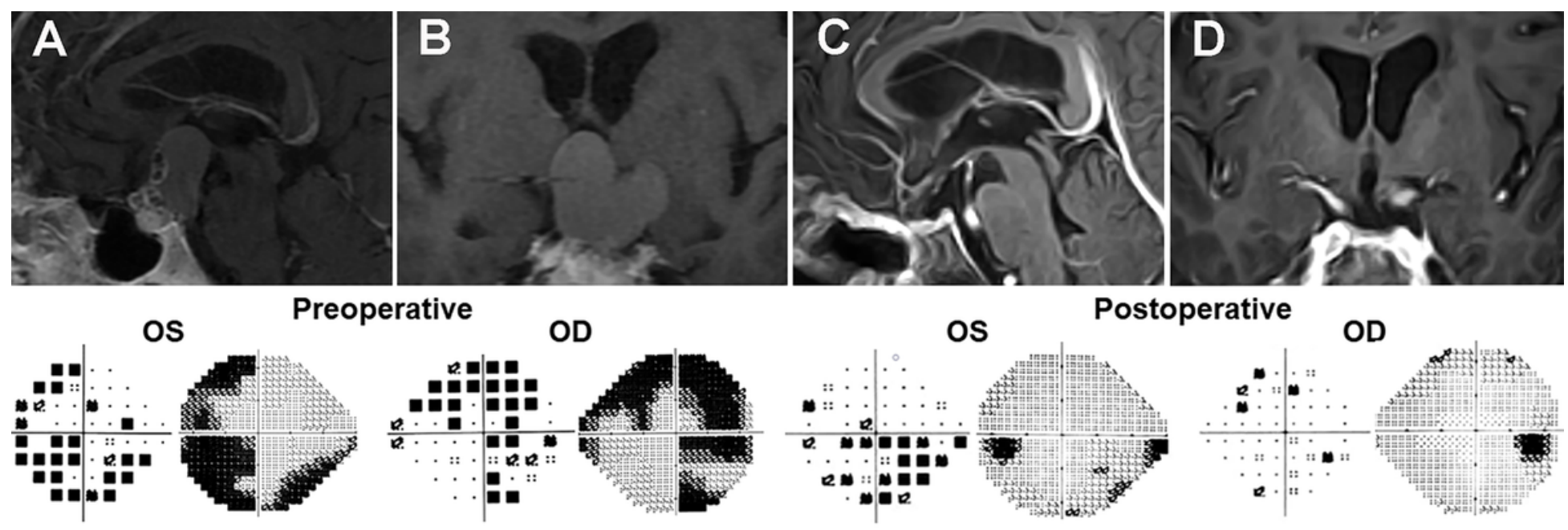

$B C V A=0.6 \quad M D=-11.36 \mathrm{~dB}$

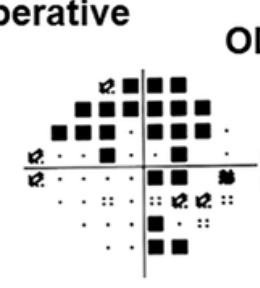

OD

Postoperative

$B C V A=0.6$

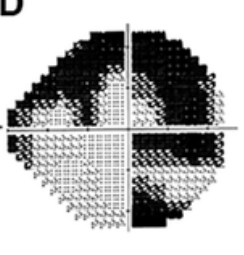

$M D=-11.91 \mathrm{~dB}$ RNFL Analysis
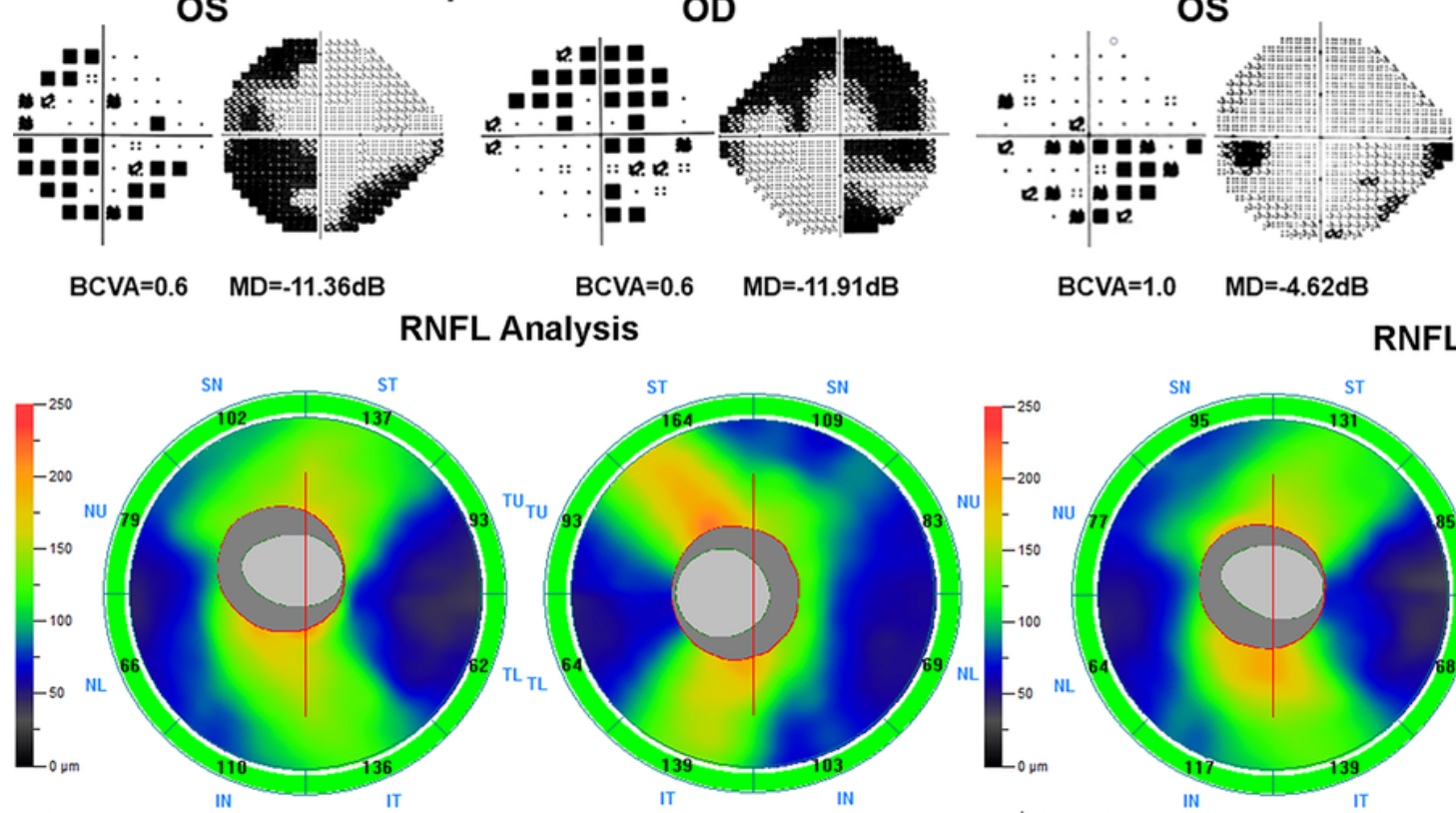

$B C V A=1.0 \quad M D=-4.62 \mathrm{~dB}$

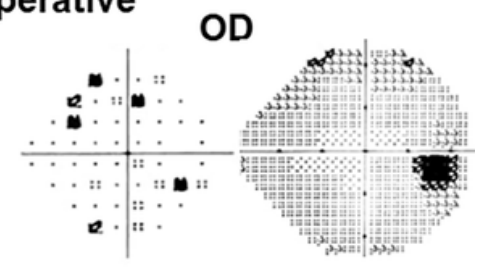

$B C V A=1.0 \quad M D=-2.74 d B$

RNFL Analysis
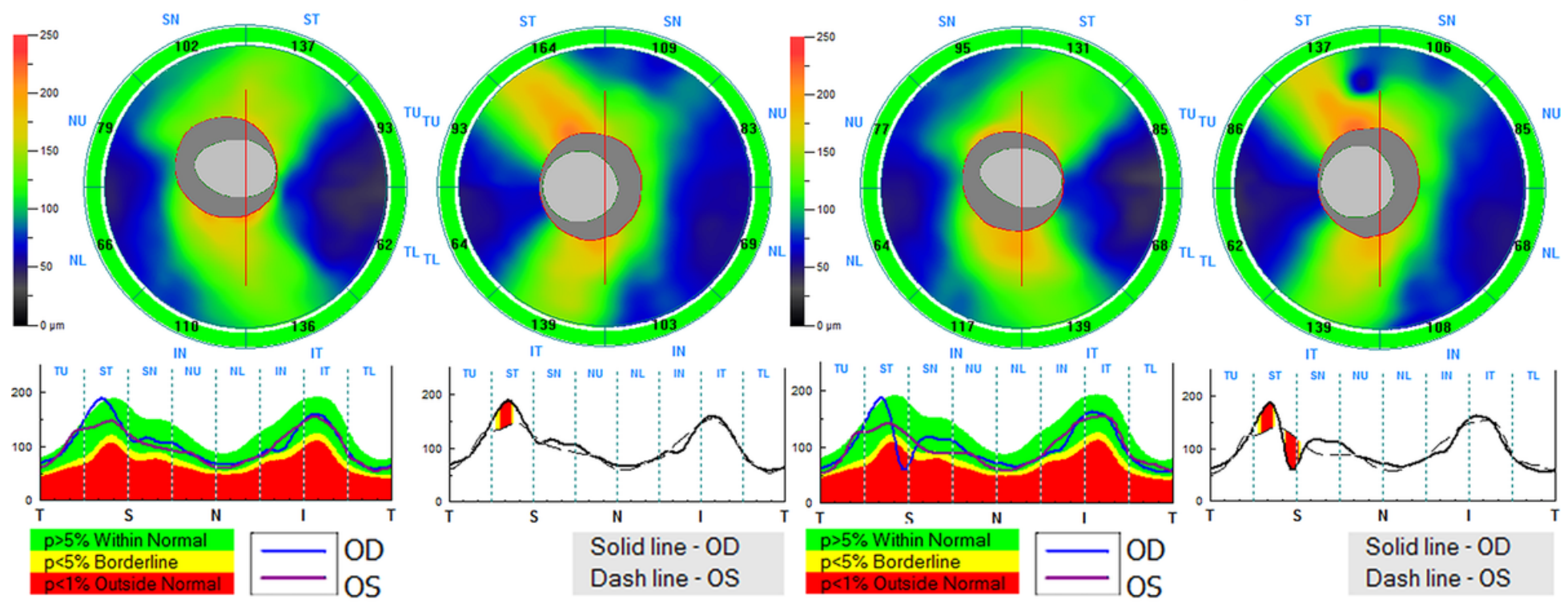

Figure 5

A 59-year-old male patient who underwent surgery for craniopharyngiomas was examined preoperatively and three months after surgery. Contrast-enhanced T1-weighted MRI scans (A, B)suggested a suprasellar cystic-solid lesion compressing downward the optic chiasma. Preoperative Humphrey visual field test showed mainly temporal visual field defects in both eyes. Preoperative OCT suggested normal cpRNFL thickness in both eyes. After total resection of the tumor (C, D), the optic nerves were sufficiently decompressed, the visual acuity and visual field in both eyes dramatically improved after surgery. $\mathrm{OCT}=$ Optical coherence tomography; $\mathrm{cpRNF=circumpapillary} \mathrm{retinal} \mathrm{nerve} \mathrm{fiber} \mathrm{layer;} B C V A=$ best corrected visual acuity; $\mathrm{MD}=$ mean deviation. 


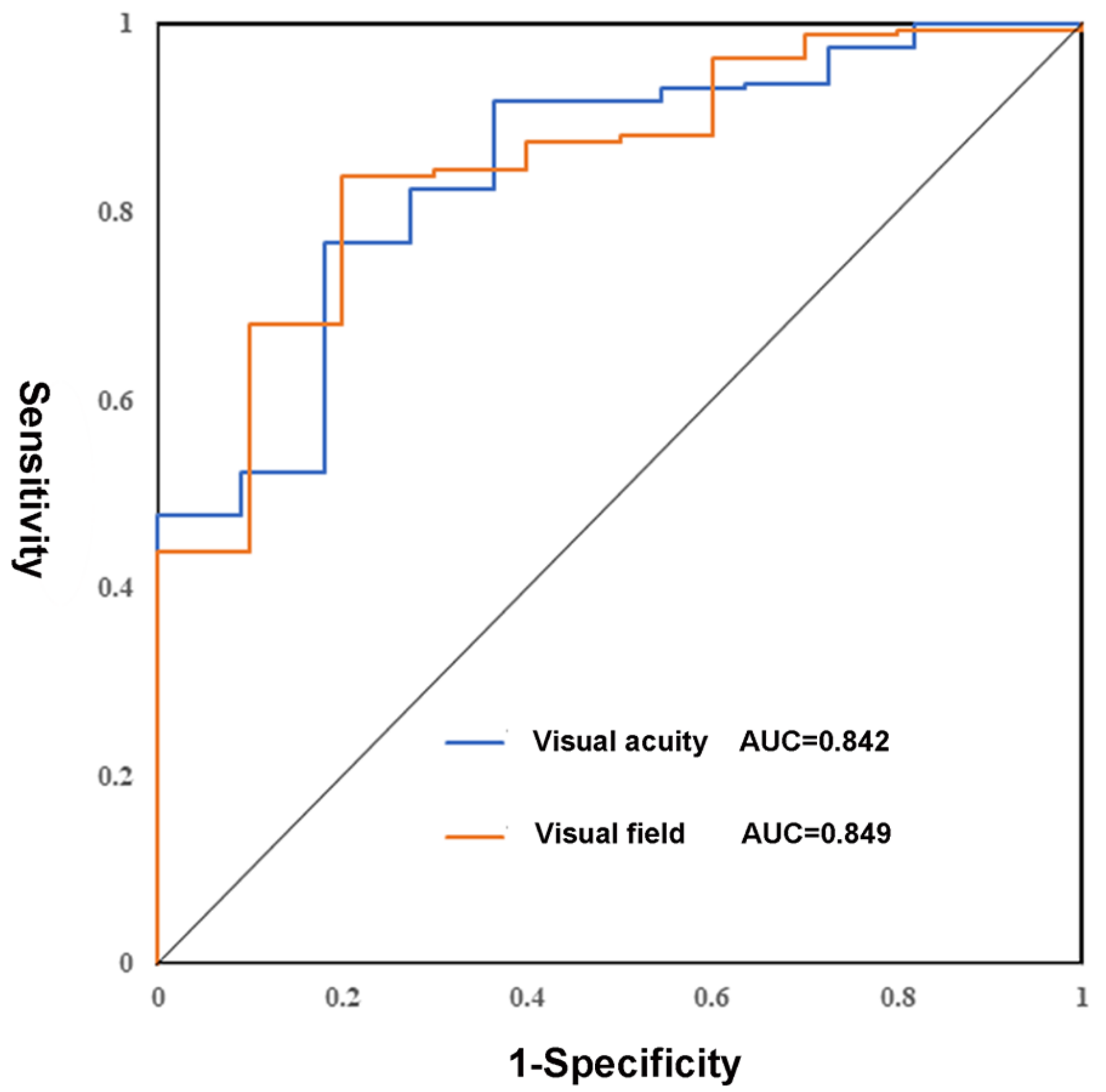

Figure 6

Receiver operating characteristic (ROC) curves for the discriminative performance of the multivariable prediction models developed for visual prognosis. 